\title{
Numerical analysis of the dynamic behaviour of a nonlinear preloaded intervertebral disc
}

\author{
J.-B. Garcher, L. Rouleau* and J.-F. Deü \\ * Laboratoire de Mécanique des structures et des Systèmes Couplés (LMSSC) \\ Conservatoire national des arts et métiers \\ 2 Rue Conté, 75003, Paris, France \\ e-mail: lucie.rouleau@lecnam.net, web page: http://www.lmssc.cnam.fr/en
}

\begin{abstract}
In order to understand the mechanisms in degenerative disc disease and support the design of robust disc prothesis, accurate modelling of the intervertebral disc's (IVD) dynamics is necessary. Many experimental studies relate changes in the mechanical properties of the disk to the degenerative state of the IVD $[1,2]$. However, further studies are necessary to better assess the influence of parameters which may affect those mechanical properties on the dynamic behaviour of the IVD, especially its dissipative behaviour. The goal of this work is to investigate the effect the static preload and the frequency of excitation through numerical simulations.
\end{abstract}

The first step consists in developing a finite element model which accounts for the complexity of the IVD (heterogeneity, fibre anisotropy, hyperelasticity, viscoelasticity). The hyperelastic Holzapfel-Gasser-Ogden (HGO) material model [3] was implemented to describe the nonlinear behaviour of the annulus fibrosus. The dissipative behaviour of the fibres is also considered through a finite strain viscoelasticity model, compatible with the hyperelastic formulation [4]. The proposed finite element model is validated with numerical results from the literature, by performing quasi-static simulations under compression loading on healthy IVD.

The second step consists in characterising the dynamic response of the IVD to a harmonic load about a nonlinear preloaded state. The influence of the preload's amplitude and the frequency of excitation on the stiffness and damping properties of the IVD are to be related with the dynamic response of the IVD.

\section{REFERENCES}

[1] Iatridis, J.C. and Setton, L.A. and Weidenbaum, M., Mow, V.C. Alterations in the mechanical behavior of the human lumbar nucleus pulposus with degeneration and aging. Journal of Orthopaedic Research (1997) 15(2):318-22.

[2] Wilson, S. and Alkalay, R. and Myers, B. Effect of the degenerative state of the intervertebral disk on the impact characteristics of human spine segments. Frontiers in Bioengineering and Biotechnology (2013), https://doi.org/10.3389/fbioe.2013.00016.

[3] Gasser, T.C. and Ogden, R.W. and Holzapfel, G.A. Hyperelastic Modelling of Arterial Layers with Distributed Collagen Fibre Orientations. Journal of The Royal Society Interface (2006) 3(6):15-35.

[4] Govindjee, S. and Simo, J.C. Mullins' Effect and the Strain Amplitude Dependence of the Storage Modulus. International Journal of Solids and Structures (1992) 29(14-15):1737-51. 